\title{
Parathyroid Imaging: The Importance of Pinhole Collimation with Both Single- and Dual-Tracer Acquisition
}

\author{
William C. Klingensmith III ${ }^{1}$, Phillip J. Koo ${ }^{1}$, Adam Summerlin ${ }^{1}$, Bradley W. Fehrenbach ${ }^{1}$, Ramesh Karki ${ }^{1}$, \\ Benjamin C. Shulman ${ }^{2}$, Christopher D. Raeburn ${ }^{3}$, and Robert C. McIntyre, Jr. ${ }^{3}$ \\ ${ }^{1}$ Division of Nuclear Medicine, Department of Radiology, University of Colorado School of Medicine, Aurora, Colorado; \\ ${ }^{2}$ Department of Biostatistics and Informatics, Colorado School of Public Health, Aurora, Colorado; and ${ }^{3}$ Division of GI, \\ Tumor, and Endocrine Surgery, Department of Surgery, University of Colorado School of Medicine, Aurora, Colorado
}

Our objective was to rigorously compare pinhole and parallel-hole collimation in an intrapatient, intrastudy design in 2 parathyroid imaging protocols: the first was dual-phase 99mTc-sestamibi imaging, and the second was dual-phase ${ }^{99 m T c-s e s t a m i b i ~ p l u s ~}$ dual-tracer ( ${ }^{99 m}$ Tc-sestamibi and $\left.{ }^{123} \mathrm{l}\right)$ simultaneous-acquisition subtraction imaging. Methods: Thirty-three patients with 37 surgically proven nonectopic parathyroid adenomas were evaluated. Anterior pinhole and parallel-hole images of the neck were

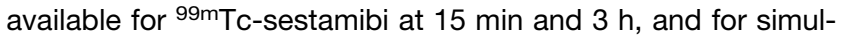
taneously acquired ${ }^{99 m} \mathrm{Tc}$-sestamibi and ${ }^{123}$ / subtraction at $15 \mathrm{~min}$, all from a single study. The images were modified so that all had a square border and so that the thyroid filled approximately three quarters of the image. The images were evaluated by 2 experienced nuclear medicine physicians who did not know the surgical results or whether the images were acquired with pinhole or parallel-hole collimation. The observers indicated the location of any identified adenoma and graded the certainty of diagnosis on a 3-point scale. Results: The localization success rate for the 2 observers combined for the single-tracer dualphase images was $66.2 \%$ with pinhole collimation and $43.2 \%$ with parallel-hole collimation $(P<0.0001)$. The localization success rate with the addition of the dual-tracer simultaneousacquisition subtraction image was $83.8 \%$ with pinhole collimation and $62.2 \%$ with parallel-hole collimation $(P=0.0018)$. In addition, the degree of certainty of localization was greater with pinhole collimation with both imaging protocols $(P<0.001$ in both cases). Conclusion: In the anterior projection, pinhole collimation is superior to parallel-hole collimation for parathyroid imaging with either dual-phase ${ }^{99 \mathrm{~m} T c-s e s t a m i b i}$ or dual-phase 99mTc-sestamibi plus dual-tracer ( ${ }^{99 \mathrm{~m} T c-s e s t a m i b i}$ and $\left.{ }^{123} \mathrm{I}\right)$ simultaneous-acquisition subtraction.

Key Words: parathyroid imaging; pinhole collimator; dual-tracer subtraction

J Nucl Med Technol 2013; 41:99-104

DOI: 10.2967/jnmt.112.118208

\footnotetext{
Received Dec. 5, 2012; revision accepted Jan. 31, 2013.

For correspondence or reprints contact: William C. Klingensmith III, Department of Radiology, School of Medicine, University of Colorado, P.O. Box L-954, Anschutz Medical Center, Aurora, CO 80113.

E-mail: william.klingensmith@ucdenver.edu

Published online Mar. 28, 2013.

COPYRIGHT @ 2013 by the Society of Nuclear Medicine and Molecular Imaging, Inc.
}

$\mathbf{T}$ he parathyroid imaging study is frequently performed for localization of parathyroid adenomas in patients with hypercalcemia and elevated serum parathormone levels (1). There are a wide variety of protocols for parathyroid imaging, but most of them include planar imaging with pinhole or parallel-hole collimation (1-9). It is well known that for small structures $\gamma$-cameras with pinhole collimation are capable of higher spatial resolution than parallel-hole collimators for basic physical reasons (10). However, there has been a trend toward substituting parallel-hole collimation for pinhole collimation (11-13).

A recent survey of Finnish hospitals indicated that only $28 \%$ used pinhole collimation for parathyroid imaging (9). In addition, a recent survey of Veterans Administration Hospitals found that only $63 \%$ of hospitals used pinhole collimation for thyroid imaging (10). Although not directly addressed, it seems likely that the hospitals that used parallel-hole collimation for thyroid imaging would also use parallel-hole collimation for parathyroid imaging. A 2004 review article on parathyroid imaging recommends parallel-hole collimation and does not mention pinhole collimation (2). This trend has been attributed to many factors including the mistaken belief that digital magnification can be substituted for physical pinhole magnification; the emphasis on SPECT and SPECT/CT, which inherently use parallel-hole collimation; and the need for cost savings - that is, some hospitals no longer purchase pinhole collimators $(1,11,13-15)$.

Previous studies have shown that pinhole collimation is superior to parallel-hole collimation in parathyroid imaging studies $(16,17)$. In the study by Tomas et al., the sensitivity for parathyroid adenoma was $89 \%$ with pinhole collimation and $56 \%$ with parallel-hole collimation, with no difference in specificity (16). In the study by Arveschoug et al., the correct identification and lateralization of parathyroid adenoma was $88 \%$ with pinhole collimation and $54 \%$ with parallelhole collimation (17). However, these previous studies used a dual-phase ${ }^{99 \mathrm{~m}}$ Tc-sestamibi protocol and did not evaluate the effect of collimator selection in conjunction with the more accurate dual-tracer $\left({ }^{99} \mathrm{~m}\right.$ Tc-sestamibi and $\left.{ }^{123} \mathrm{I}\right)$ simultaneous-acquisition subtraction protocol $(1,18)$. It is possible that perfectly coregistered subtraction images generated 
from images acquired with parallel-hole collimation may compensate for the lack of high-resolution pinhole images.

We compared the ability of pinhole and parallel-hole images in the anterior projection from a dual-phase ${ }^{99 \mathrm{~m} T c-s e s t a m i b i}$ protocol to detect and localize parathyroid adenomas relative to the thyroid lobes without and with simultaneously acquired ${ }^{99 \mathrm{~m}} \mathrm{Tc}$-sestamibi and ${ }^{123} \mathrm{I}$ subtraction images. Both sets of images were obtained from a single study in each patient so that the comparisons were performed on an intrapatient and intrastudy basis. In addition, the images were processed so that the observers could not determine the type of collimation from the shape of the images or the magnification of the thyroid.

\section{MATERIALS AND METHODS}

\section{Patient Database}

The parathyroid imaging studies were derived from a previously published series of 37 consecutive patients with surgically proven parathyroid adenomas (18). Four of the patients in the previous series had ectopic adenomas and were excluded because the adenomas were outside the field of view of the pinhole image. No patients were eliminated because they had had previous parathyroid or thyroid surgery or were taking thyroid replacement hormone. The study was approved by the Colorado Multiple Institutional Review Board.

\section{Imaging Protocol}

Patients were initially given $22.2 \mathrm{MBq}(600 \mu \mathrm{Ci})$ of ${ }^{123}$ I orally. Two hours later, the patients were given $925 \mathrm{MBq}(25 \mathrm{mCi})$ of

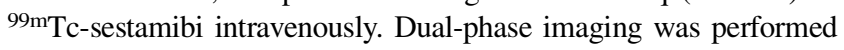
at $15 \mathrm{~min}$ and at $3 \mathrm{~h}$ after administration of the ${ }^{99 \mathrm{~m}} \mathrm{Tc}$-sestamibi. The images were acquired with a dual-head e.cam $\gamma$-camera with an energy resolution of $9 \%$ (Siemens Medical Solutions). The camera was configured with a pinhole collimator on one head and a low-energy high-resolution collimator on the other head. At each time, 5-min pinhole images of the neck in the anterior and both anterior oblique projections $\left(45^{\circ}\right)$ were obtained with a $4-$ $\mathrm{mm}$ pinhole insert. In addition, at both times a 5-min low-energy high-resolution parallel-hole collimator image with a field of view extending from the submandibular glands to the left ventricle was obtained in the anterior projection.

The ${ }^{99 \mathrm{~m}} \mathrm{Tc}-$ sestamibi and ${ }^{123} \mathrm{I}$ images were acquired simultaneously with a $12 \%$ energy window centered at $140 \mathrm{keV}$ for ${ }^{99 \mathrm{~m}} \mathrm{Tc}$-sestamibi and an $8 \%$ energy window centered at $159 \mathrm{keV}$ for ${ }^{123} \mathrm{I}(3)$. There was no correction for cross talk $(1,16)$. Subtraction images were constructed for all pairs of ${ }^{99 \mathrm{~m}} \mathrm{Tc}$-sestamibi and ${ }^{123} \mathrm{I}$ pinhole images. The subtractions were done manually by increasing the amount of subtraction of the ${ }^{123}$ I thyroid image from the ${ }^{99 m}$ Tc-sestamibi image until the thyroid was essentially no longer visible.

\section{Image Processing}

Because, in our routine protocol, the parallel-hole collimator image is obtained in only the anterior projection, only the anterior pinhole image was used in all comparisons. Also, in our routine protocol anterior parallel-hole images are obtained for both ${ }^{99 \mathrm{~m}} \mathrm{Tc}-$ sestamibi and ${ }^{123} \mathrm{I}$ but no subtraction image is generated. For the purposes of this study, an anterior parallel-hole subtraction image, ${ }^{99 \mathrm{~m} T c}$-sestamibi minus ${ }^{123} \mathrm{I}$, was generated at $15 \mathrm{~min}$ only for each patient study. All subtractions were done manually. The algorithm

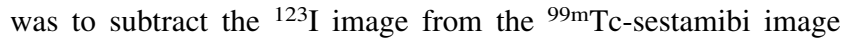
until the thyroid was just removed, leaving a salt-and-pepper appearance to the thyroid bed. Each of the pinhole and parallel-hole collimator images was processed so that the field of view was square and so that the thyroid filled approximately three quarters of the field of view.

\section{Image Sets}

The images from each patient study were used to create 4 image sets. The first image set consisted of anterior images of ${ }^{99 \mathrm{~m}} \mathrm{Tc}$-sestamibi at $15 \mathrm{~min}$ and at $3 \mathrm{~h}$ acquired with a pinhole collimator (Figs. 1 and 2). The second image set was the same as the first except that the images were acquired with a parallel-hole collimator. The third and fourth image sets consisted of the first 2 image sets plus the corresponding pinhole or parallel-hole collimator anterior subtraction image (Figs. 1 and 2).

FIGURE 1. Pinhole collimator (A) and parallel-hole collimator (B) images of $99 \mathrm{mTc}-$ sestamibi at $15 \mathrm{~min}$ and $3 \mathrm{~h}$, and image of $99 \mathrm{~m} T c$-sestamibi minus 123 at $15 \mathrm{~min}$, in patient with hyperparathyroidism and 380-mg parathyroid adenoma located inferiorly on right. With pinhole collimation, 2 observers correctly localized adenoma without and with subtraction image with degree-of-certainty grades of 2 and 3 for one observer and 3 and 3 for the other. With parallel-hole collimation, observers did not identify adenoma without subtraction image and did localize adenoma with subtraction image with grades of 1 and 1 .

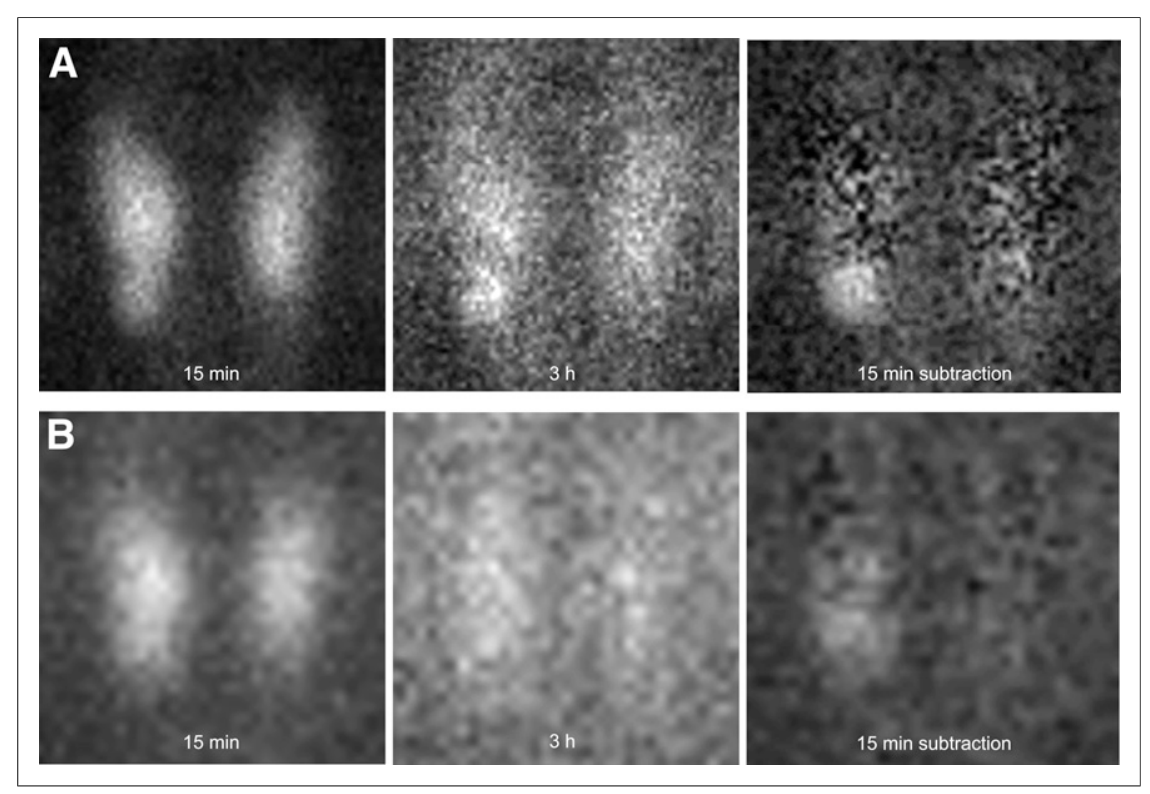


Two groups of images were created for interpretation. The first group was created by combining the first and second image sets, single-tracer dual-phase (ST-DP), with either pinhole or parallelhole collimation in random order. The second group was created by combining the third and fourth image sets, single-tracer dualphase plus subtraction (ST-DP $+\mathrm{S}$ ), with either pinhole or parallel-hole collimation in random order. Thus, each patient was present twice in each of the 2 image interpretation groups, once with pinhole images and once with parallel-hole images (Figs. 1 and 2).

\section{Image Interpretation}

Two experienced nuclear medicine physicians independently interpreted the 2 groups, first the ST-DP image set and then the ST-DP $+\mathrm{S}$ image set. The observers were masked to all patient identification and to the type of collimation used for image acquisition. There was at least 1 mo between interpretations of the 2 groups for both physicians.

The images were interpreted on a 4-point scale, with zero indicating no evidence of a parathyroid adenoma and 1 through 3 indicating a focus of activity consistent with a parathyroid adenoma. A grade of 1 indicated a possible parathyroid adenoma, 2 a probable parathyroid adenoma, and 3 a definite parathyroid adenoma. In addition to the certainty of a parathyroid adenoma, the interpreting physician indicated the location of the abnormality with respect to side (right or left) and location with respect to the thyroid lobes (superior, middle, or inferior to the thyroid). If a patient's images showed more than one parathyroid abnormality, the interpretation process was repeated for each abnormality beginning with the most obvious abnormality. If an observer identified an incorrect location (false-positive) and did not grade the correct location, the correct location was assigned a 0 .

\section{Surgery}

All operations were performed by 1 of 2 endocrine surgeons and 2 otolaryngologic surgeons. Intraoperative assays of serum parathormone were done at the beginning of surgery and after the removal of each parathyroid gland. An intraoperative decrease in blood parathormone level of at least $50 \%$ was considered evidence of curative surgery. The surgical finding, including intraoperative parathormone values, and the histopathologic findings were used as diagnostic proof.

\section{Pathologic Diagnosis}

The diagnosis was based primarily on the presence of hypercellularity.

\section{Statistical Analysis}

Sensitivity, the primary outcome, was defined as the proportion of locations of parathyroid adenomas that were correctly identified. The site location was coded as correct if the observer's response matched the surgical result exactly or within half the distance between the inferior and superior pole to allow for variation in the accuracy of the surgical description. Thus, an imaging location of middle thyroid was considered to match a surgical location of superior or inferior and vice versa. The number of times a middle location by imaging was counted as correct in comparison to a superior or inferior location by surgery and vice versa was quantified for each observer.

Specificity is difficult to calculate because the observers knew that each patient had at least one adenoma. In addition, it is considered good practice in the interpretation of parathyroid imaging studies to mention possible secondary adenomas in case there are multiple adenomas (1). At the time of surgery, if the serum parathormone level does not decrease by $50 \%$ after removal of the first adenoma, the surgeon then has a guide to the location of a likely second adenoma. However, the number of false-positive localizations was determined for each observer for pinhole and parallel collimation with and without subtraction images and served as an indicator of specificity.

The sensitivity of the 4 protocols was compared statistically using a pseudo-likelihood estimate for the 6 pairwise differences between the 4 protocols (parallel and pinhole methods, each with and without subtraction) in a repeated-measures model for the binary outcome (19). Observer and protocol were entered as main effects; patient was designated as a repeated effect. Descriptive statistics for the number of lesions located, the sensitivity rate, and its $95 \%$ exact binomial confidence interval were also computed. A similar model was used to evaluate the certainty score $(0,1,2$, or 3$)$
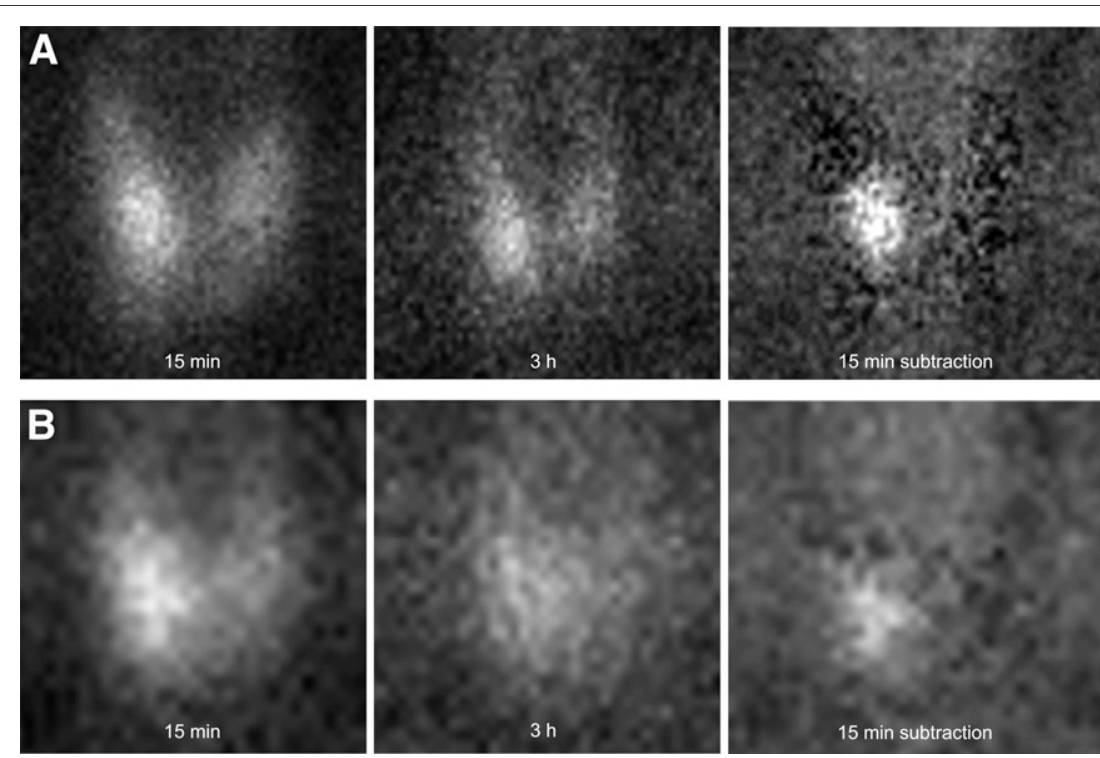

FIGURE 2. Pinhole collimator (A) and parallel-hole collimator (B) images of 99mTc-sestamibi at $15 \mathrm{~min}$ and $3 \mathrm{~h}$, and image of $99 \mathrm{mTc}$-sestamibi minus ${ }^{123}$ at $15 \mathrm{~min}$, in patient with hyperparathyroidism and 1,010-mg parathyroid adenoma located inferiorly on right. With pinhole collimation, 2 observers correctly localized adenoma without and with subtraction image with degree-of-certainty grades of 3 and 2 for one observer and 3 and 3 for the other. With subtraction image, one observer located adenoma at middle level whereas other localizations were all inferior. With parallel-hole collimation, only one observer identified adenoma without subtraction image, grades of 0 and 1 , and both observers localized adenoma with subtraction image with grades of 1 and 1 . 
in a multinomial model for the ordered responses (19). Again, observer and protocol were entered as main effects; patient was designated as a repeated effect.

\section{RESULTS}

\section{Patient Data}

Thirty-three patients with one or more surgically proven adenomas who underwent preoperative parathyroid imaging formed the study database. All patients had primary hyperparathyroidism. There were 25 women and 8 men. The average age for women was $56.8 \mathrm{y}$ (range, 36-70 y), and for men it was $55.8 \mathrm{y}$ (range, 34-81 y). Four of the 33 patients had undergone previous unsuccessful surgery for a parathyroid adenoma or a partial thyroidectomy, and 3 patients were taking replacement thyroxine at the time of imaging. For all 33 patients, the average blood calcium level was $10.8 \mathrm{mg} / \mathrm{dL}$ (range, $9.6-12.3 \mathrm{mg} / \mathrm{dL}$ ) and the average serum parathormone level, at the time of surgery, was $136.7 \mathrm{pg} / \mathrm{mL}$ (range, 50-259 pg/mL). The reference ranges for blood calcium and parathormone levels at our institution are $8.5-10.3 \mathrm{mg} / \mathrm{dL}$ and $12-88 \mathrm{pg} / \mathrm{mL}$, respectively.

\section{Surgical Results}

Thirty patients had 1 parathyroid adenoma, 2 patients had 2 adenomas, and 1 patient had 3 adenomas for a total of 37 adenomas, all in the region of the thyroid. The average intraoperative decrease in serum parathormone level after resection of all adenomas was 77\% (range, 52\%-95\%).

\section{Pathologic Findings}

Histology confirmed hypercellular parathyroid tissue in all resected glands. The average weight of the adenomas was $689 \mathrm{mg}$ (range, 60-4,130 mg).

\section{Protocol Analysis}

The interpretation results on a per-observer and peradenoma basis are shown in Table 1. In the evaluation of the single-tracer dual-phase ${ }^{99 \mathrm{~m}} \mathrm{Tc}$-sestamibi group, observer

TABLE 1

Grading Results for Each of 4 Protocols for 37 Adenomas in 33 Patients

\begin{tabular}{lcrccc}
\hline & & \multicolumn{4}{c}{$\begin{array}{c}\text { Degree of certainty of } \\
\text { location }\end{array}$} \\
\cline { 3 - 6 } Protocol & Observer & 0 & 1 & 2 & 3 \\
\hline ST-DP & A & 19 & 7 & 6 & 5 \\
Pinhole & B & 6 & $16(9)$ & $8(1)$ & 7 \\
ST-DP & A & 25 & $5(1)$ & 4 & 3 \\
Parallel & B & 17 & $8(7)$ & 8 & 4 \\
ST-DP + S & A & 8 & $11(2)$ & $6(3)$ & $12(2)$ \\
Pinhole & B & 4 & $13(7)$ & 10 & 10 \\
ST-DP + S & A & 16 & $7(1)$ & $7(1)$ & $7(1)$ \\
Parallel & B & 12 & $12(2)$ & 9 & 4 \\
& & & & & 4
\end{tabular}

${ }^{\dagger} 0=$ no adenoma seen; 1 = possible adenoma; 2 = probable adenoma; 3 = definite adenoma.

Numbers in parentheses indicate number of localizations that were incorrect.
TABLE 2

Localization Success for Each of 4 Protocols

\begin{tabular}{lcccc}
\hline & \multicolumn{4}{c}{ Localization success } \\
\cline { 3 - 5 } Protocol & Observer & Adenomas & Correct & $95 \% \mathrm{Cl}$ \\
\hline ST-DP & $\mathrm{A}$ & $18 / 37$ & 0.49 & $0.32-0.66$ \\
Pinhole & $\mathrm{B}$ & $31 / 37$ & 0.84 & $0.68-0.94$ \\
ST-DP & $\mathrm{A}$ & $12 / 37$ & 0.32 & $0.18-0.50$ \\
Parallel & $\mathrm{B}$ & $20 / 37$ & 0.54 & $0.37-0.71$ \\
ST-DP $+\mathrm{S}$ & $\mathrm{A}$ & $29 / 37$ & 0.78 & $0.62-0.90$ \\
Pinhole & $\mathrm{B}$ & $33 / 37$ & 0.89 & $0.75-0.97$ \\
ST-DP $+\mathrm{S}$ & $\mathrm{A}$ & $21 / 37$ & 0.57 & $0.39-0.73$ \\
Parallel & $\mathrm{B}$ & $25 / 37$ & 0.68 & $0.50-0.82$ \\
& \multicolumn{5}{c}{} & \\
\hline Cl $=$ exact binomial confidence interval. \\
There were 37 adenomas in 33 patients. \\
\hline
\end{tabular}

A tended to have a lower sensitivity and lower false-positive rate (higher specificity) than observer $B$. This difference was not present when the ${ }^{99 \mathrm{~m}}$ Tc-sestamibi-minus- ${ }^{123}$ I image was included. The interpretation results on a per-observer and per-adenoma basis when degree of certainty grades 1 through 3 are combined are shown in Table 2.

For both observers combined and any degree of certainty, the localization success rate for the single-tracer dual-phase image set with pinhole collimation was $66.2 \%$, and with parallel-hole collimation it was $43.2 \%(P<0.0001)$ (Fig. 3). Likewise, the localization success rate with the addition of the dual-tracer simultaneous-acquisition subtraction image with pinhole collimation was $83.8 \%$, and with parallel-hole collimation it was $62.2 \%(P=0.0018)$. The addition of the dual-tracer subtraction images significantly improved the localization success rate for both pinhole and parallel-hole collimation $(P=0.0039$ and $P=0.0085$, respectively). In addition, the degree of certainty of localization was greater with pinhole collimation both without and with the subtraction image $(P=0.0003$ and $P<0.0001$, respectively).

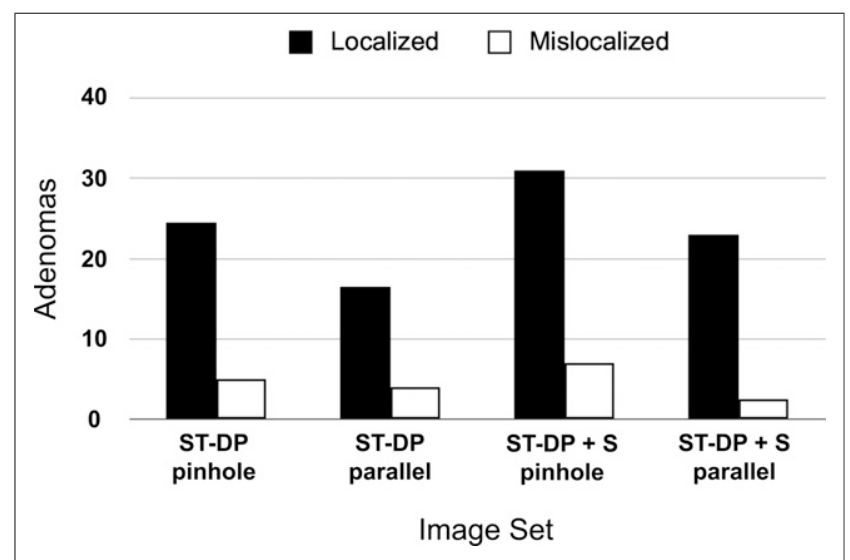

FIGURE 3. Performance for each of 4 protocols is shown for correctly localizing position of 37 parathyroid adenomas in 33 patients. Data represent average results for 2 observers. 
For both observers combined, the number of adenoma mislocalizations for the single-tracer dual-phase image set was similar for pinhole and parallel-hole collimation (Table 1; Fig. 3). The number of adenoma mislocalizations for the singletracer dual-phase image plus the dual-tracer simultaneousacquisition subtraction image was somewhat higher for pinhole collimation than for parallel-hole collimation.

In patients with multiple adenomas, the nonprimary less obvious adenomas accounted for a disproportionate number of undetected adenomas. For the most accurate protocolsingle-tracer dual-phase with pinhole collimation plus dualtracer subtraction-one observer detected none of the 4 nonprimary adenomas and the other observer detected 2 of the 4 nonprimary adenomas with grades of 1: a combined localization success rate of $25 \%$. In comparison, the average success rate of the 2 observers was $83.5 \%$ for the 33 primary adenomas.

Four of the 37 adenomas were given a middle location relative to the thyroid lobe at surgery $(10.8 \%)$. Observer A used the middle location in 15 of 91 localizations (16.5\%), and observer $\mathrm{B}$ used the middle location in 21 of 135 localizations (15.6\%), for a total of 36 middle localizations. For both observers, 10 of the middle localizations matched middle localizations at surgery and 6 of the middle localizations were false-positives. The remaining 20 middle localizations were on the same side as the adenoma that was located as superior or inferior at surgery; these were counted as correct.

\section{DISCUSSION}

This study demonstrated the superiority of pinhole collimation in comparison to parallel-hole collimation in the detection and localization of parathyroid adenomas in patients with hyperparathyroidism. This finding was true both for the commonly used single-tracer dual-phase ${ }^{99 \mathrm{~m}} \mathrm{Tc}$-sestamibi protocol and for the more accurate dual-tracer ${ }^{99 \mathrm{~m}} \mathrm{Tc}$-sestamibi and ${ }^{123}$ I) simultaneous-acquisition subtraction protocol $(1,18)$. Pinhole and parallel-hole collimators were compared in the anterior projection because only an anterior parallel-hole collimator image was part of our standard protocol. The advantage of pinhole over parallel-hole collimation in the anterior oblique projections compared with the anterior projection would likely be the same or greater because the parallel-hole collimator would need to be even farther from the anterior neck in the oblique projections in order to clear the patient's shoulders.

This study also demonstrated that the addition of a dualtracer $\left({ }^{99 \mathrm{~m}} \mathrm{Tc}-\right.$ sestamibi and $\left.{ }^{123} \mathrm{I}\right)$ simultaneous-acquisition subtraction image increases the sensitivity for parathyroid adenoma localization for both pinhole and parallel-hole collimation. However, the sensitivity for localization of parathyroid adenomas for the full protocol, from which the anterior images in this study were obtained, is significantly higher, at $94 \%$ (18).

There have been several reports of the use of dual tracers $\left({ }^{99 \mathrm{~m}} \mathrm{Tc}\right.$-sestamibi and ${ }^{123} \mathrm{I}$ ) with simultaneous-acquisition subtraction for parathyroid imaging using SPECT or SPECT/CT
$(1,20-23)$. Pinhole imaging in the anterior and anterior oblique projections has been shown to be superior to SPECT imaging alone without planar imaging using the singletracer dual-phase protocol (3). However, there is no evidence that dual-tracer simultaneous-acquisition subtraction SPECT/CT without planar pinhole imaging is equal to dual-tracer simultaneous-acquisition subtraction SPECT/CT with planar pinhole imaging. Our current routine protocol uses single-phase dual-tracer ${ }^{99 \mathrm{~m}} \mathrm{Tc}$-sestamibi and $\left.{ }^{123} \mathrm{I}\right) \mathrm{si}$ multaneous-acquisition subtraction SPECT/CT in addition to planar pinhole imaging. The disadvantage of eliminating planar pinhole imaging in favor of SPECT/CT alone will be the subject of a future report.

Previous studies have shown that pinhole collimation is superior to parallel-hole collimation in parathyroid imaging, but they used the single-tracer dual-phase ${ }^{99 \mathrm{~m}} \mathrm{Tc}$-sestamibi protocol rather than the more accurate single-phase dualtracer $\left({ }^{99 \mathrm{~m}} \mathrm{Tc}\right.$-sestamibi and $\left.{ }^{123} \mathrm{I}\right)$ simultaneous-acquisition subtraction protocol $(16,17)$. Thus, it was possible that perfectly coregistered subtraction images generated from images acquired with parallel-hole collimation would compensate for the lack of high-resolution pinhole images. In addition, the previous studies did not normalize the images relative to image shape and thyroid size. In the present study, the images were processed so that the shape of the images and the size of the thyroid were the same for pinhole and parallelhole collimation in order to eliminate visual cues that might bias the results.

\section{CONCLUSION}

Although pinhole collimation was used in the original $\gamma$-camera and is recognized as having spatial resolution superior to that of parallel-hole collimation, it has been replaced by parallel-hole collimation in a significant number of institutions for parathyroid imaging. The present intrapatient, intrastudy comparison demonstrated the superiority of pinhole collimation relative to parallel-hole collimation for localization of parathyroid adenomas both in the conventional single-tracer dual-phase ${ }^{99 \mathrm{~m}} \mathrm{Tc}$-sestamibi protocol and in the more accurate dual-tracer ${ }^{99 \mathrm{~m}} \mathrm{Tc}$-sestamibi and ${ }^{123}$ I) simultaneous-acquisition subtraction protocol. The results suggest that planar imaging for parathyroid adenomas should be performed with pinhole collimation.

\section{DISCLOSURE}

This work was partially funded by the Talofa Foundation, Denver, Colorado, and by NIH/NCRR Colorado CTSI grant UL1 RR025780. No other potential conflict of interest relevant to this article was reported.

\section{REFERENCES}

1. Mullan BP. Nuclear medicine imaging of the parathyroid. Otolaryngol Clin North Am. 2004;37:909-939.

2. Palestro CJ, Tomas MB, Tronco GG. Radionuclide imaging of the parathyroid glands. Semin Nucl Med. 2005;35:266-276.

3. Ho Shon IA, Yan W, Roach P, et al. Comparison of pinhole and SPECT Tc-99mMIBI imaging in primary hyperparathyroidism. Nucl Med Commun. 2008;29:949-955. 
4. Nichols KJ, Tomas MB, Tronco GG, et al. Preoperative parathyroid scintigraphic lesion localization: accuracy of various types of readings. Radiology. 2008;248:221-232.

5. Smith JR, Oates ME. Radionuclide imaging of the parathyroid glands: patterns, pearls and pitfalls. Radiographics. 2004;24:1101-1115.

6. Hindié E, Ugur O, Fuster D, et al. EANM parathyroid guidelines. Eur J Nucl Med Mol Imaging. 2009;36:1201-1216.

7. Ruda JM, Hollenbeak C, Stack BC. A systematic review of the diagnosis and treatment of primary hyperparathyroidism from 1995 to 2003. Otolaryngol Head Neck Surg. 2005;132:359-372.

8. Lavely WC, Goetze S, Friedman KP, et al. Comparison of SPECT/CT, SPECT, and planar imaging with single- and dual-phase ${ }^{99 \mathrm{~m}} \mathrm{Tc}$-sestamibi parathyroid scintigraphy. J Nucl Med. 2007;48:1084-1089.

9. Greenspan BS, Dillehay G, Intenzo C, et al. SNM practice guideline for parathyroid scintigraphy 4.0. J Nucl Med Technol. 2012;40:111-118.

10. Peterson TE, Shokouhi S. Advances in preclinical SPECT instrumentation. J Nucl Med. 2012;53:841-844.

11. Tunninen V, Kauppinen T, Eskola H, et al. Variable imaging and processing protocols of parathyroid scintigraphy in Finland [abstract]. Eur J Nucl Med Mol Imaging. 2009;36(suppl):S195.

12. Friedman K, Goel S, Christian P, et al. Forgotten knowledge? Current practice patterns of thyroid scintigraphy [abstract]. J Nucl Med. 2009;50(suppl):265P.

13. Rink T, Schroth HJ, Holle LH, et al. Limited sensitivity of parathyroid imaging with ${ }^{99 \mathrm{~m}} \mathrm{Tc}$-sestamibi/ ${ }^{123} \mathrm{I}$-subtraction in an endemic goiter area. J Nucl Med. 2002;43:1175-1180.

14. Thomas DL, Bartel T, Menda Y, et al. Single photon emission computed tomography (SPECT) should be routinely performed for the detection of parathyroid abnormalities utilizing technetium-99m sestamibi parathyroid scintigraphy. Clin Nucl Med. 2009;34:651-655.
15. Poullias X, Guernou M, Hapdey S, et al. ${ }^{99 m}$ Tc-sestamibi/ ${ }^{123}$ I-subtraction SPECT-CT: Interest of hybrid imaging in patients with hyperparathyroidism and candidate to surgery [abstract]. J Nucl Med. 2010;51(suppl):57P.

16. Tomas MB, Pugliese PV, Tronco GG, et al. Pinhole versus parallel-hole collimators for parathyroid imaging: an intraindividual comparison. $\mathrm{J} \mathrm{Nucl} \mathrm{Med}$ Technol. 2008;36:189-194.

17. Arveschoug AK, Bertelsen H, Vammen AB. Presurgical localization of abnormal parathyroid glands using a single injection of Tc-99m sestamibi: comparison of high resolution parallel hole and pinhole collimators, and interobserver and intraobserver variation. Clin Nucl Med. 2002;27:249-254.

18. Caveny SA, Klingensmith WC, Martin WE, et al. Parathyroid imaging: the importance of dual radiopharmaceutical simultaneous acquisition with Tc-99msestamibi and I-123. J Nucl Med Technol. 2012;40:104-110.

19. Liang KY, Zeger SL. Longitudinal data analysis using generalized linear models. Biometrika. 1986;73:13-22.

20. Neumann DR, Esselstyn CB Jr, Go RT, Wong CO, Rice TW, Obuchowski NA. Comparison of double phase ${ }^{99 \mathrm{~m}} \mathrm{Tc}$-sestamibi with ${ }^{123} \mathrm{I}-{ }^{99 \mathrm{~m}} \mathrm{Tc}$-sestamibi subtraction SPECT in hyperparathyroidism. AJR 1997;169:1671-1674.

21. Neumann DR, Obuchowski NA, DiFilippo FP. Preoperative ${ }^{123} \mathrm{I}-/{ }^{99 m} \mathrm{Tc}$-sestamibi subtraction SPECT and SPECT-CT in primary hyperparathyroidism. $\mathrm{J} \mathrm{Nucl}$ Med. 2008;49:2012-2017.

22. Heiba S, Rivera J, Jiang M, et al. The optimized evaluation of primary hyperparathyroid patients (PHP) with dual tracer (DT) neck pinhole (PH) and SPECT/CT imaging [abstract]. J Nucl Med. 2010;51(suppl 2):321P.

23. Low C, Notghi A, Pandit M, et al. Clinical outcome of SPECT-CT dual isotope (I-123/Tc-99m-MIBI) subtraction for imaging parathyroid adenoma: how good is it? [abstract]. Eur J Nucl Med Mol Imaging. 2011;38(suppl): S167. 